\title{
SOLO VOICE DETECTION VIA OPTIMAL CANCELATION
}

\author{
Christine Smit and Daniel P.W. Ellis* \\ LabROSA, Electrical Engineering \\ Columbia University \\ New York NY 10025 USA \\ \{csmit, dpwe\}@ee.columbia.edu
}

\begin{abstract}
Automatically identifying sections of solo voices or instruments within a large corpus of music recordings would be useful e.g. to construct a library of isolated instruments to train signal models. We consider several ways to identify these sections, including a baseline classifier trained on conventional speech features. Our best results, achieving frame level precision and recall of around $70 \%$, come from an approach that attempts to track the local periodicity of an assumed solo musical voice, then classifies the segment as a genuine solo or not on the basis of what proportion of the energy can be canceled by a comb filter constructed to remove just that periodicity. This optimal cancelation filter has other applications in pitch tracking and separating periodic and aperiodic energy.
\end{abstract}

\section{INTRODUCTION}

This work is motivated by a project to model the statistics of professional singers' voices, for which we would like to assemble a large collection of solo voice recordings. Many existing music recordings will contain some solo voice, but manually marking the solo passages will severely limit the amount of data we can obtain. An automatic system for identifying stretches of solo voice would allow us to mine large online music audio archives to obtain essentially unlimited quantities of solo voice or other solo instruments. Finding these "unobstructed" views of musical instruments is valuable for many applications of modeling single voices e.g. to be able to recognize them better in the context of other instruments (e.g. [1]).

Our approach is based on the idea that a solo musical passage will for the most part consist of a single note (pitch) sounding at any time. The spectral structure of an isolated pitch is characteristically simple, consisting of well-defined, regularly spaced harmonic spectral peaks(as illustrated in the top pane of figure 1) and this should allow us to distinguish these frames from either multiple simultaneous voices (middle pane) which exhibit a much more complex pattern of superimposed and interacting harmonic series, or silent gaps (bottom pane) which reveal a frequency-dependent noise floor.

We considered several different approaches. Our baseline system adopts the same approach used e.g. for detecting when a

*This work was supported by the Columbia Academic Quality Fund, and by the National Science Foundation (NSF) via a fellowship and Grants No. IIS-0238301 and IIS-0535168. Any opinions, findings and conclusions or recommendations expressed in this material are those of the authors and do not necessarily reflect the views of the NSF.
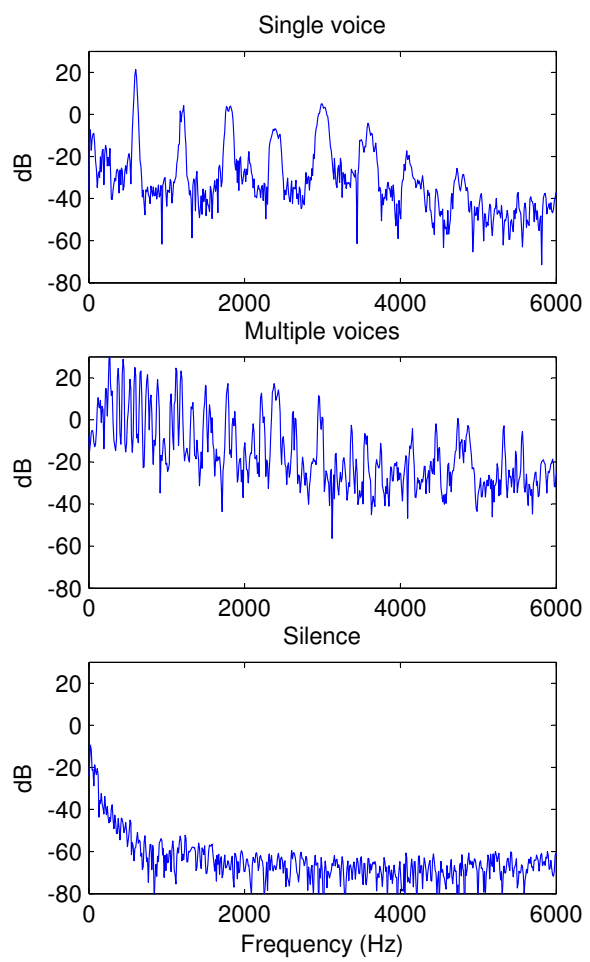

Figure 1: Example spectra taken from solo voice (top pane), ensemble accompaniment (middle pane), and background silence (bottom pane).

singer is active during accompanied music [2] by training a classifier on the ubiquitous Mel-frequency cepstral coefficients (MFCCs) borrowed from speech recognition.

We were also interested in seeing if the specific structural details visible in figure 1 could be employed directly. Our first idea was to attempt to spot the 'gaps' between the harmonics of the solo voice which might be expected to revert to the noise floor. However, we found it difficult to make this approach work, particularly as the voice pitch becomes lower and the 'gaps' become smaller.

Our most successful approach is based on the idea of attempting to model a short-time frame of the signal as consisting of a single periodicity, canceling energy at that period with an appro- 
priate comb filter (i.e. subtracting the signal delayed by one period from itself), then seeing what proportion of the total signal energy is removed. When the signal consists largely or wholly of a single periodicity, it should be possible to cancel virtually all of the periodic (tonal) energy, leading to a very large drop in energy after the filter.

In general, however, the optimal period will not be an integer number of samples, so a fractional-delay filter is required. The next section describes our approach to finding this filter, then section 3 describes our experiments with this detector, comparing it to our MFCC-based baseline. Section 4 concludes with a discussion of other uses of this optimal cancelation strategy including pitch tracking and periodic/aperiodic separation.

\section{OPTIMAL PERIODICITY CANCELATION}

By definition, a single voice has a single pitch (in the sense of a fundamental frequency), which, for musical voices, will often be relatively stationary. To detect if only a single voice was present, our approach was to find the best-fitting single period, cancel its energy, and see how completely that removed the energy of the frame. Solo voices would have only their aperiodic energy left, resulting in a large drop in energy. Polyphonies consisting of several instruments playing different pitches will have canceled only one of the periodicities, leading to a much smaller drop in energy.

After breaking up our soundfiles into $93 \mathrm{~ms}$ frames (i.e. 4096 samples at $44.1 \mathrm{kHz}$ sampling rate), we used autocorrelation to obtain an initial estimate, $\tau$, of the dominant fundamental period for each frame by finding the largest peak in the autocorrelation in the allowable fundamental frequency range of $80-800 \mathrm{~Hz}(55-551$ samples at $44.1 \mathrm{kHz}$ ). A simple filter (figure 2, top) might then be able to remove that frequency and all its harmonics:

$$
\epsilon[n]=x[n]-x[n-\tau] .
$$

If $\tau$ exactly matches the period of a purely periodic waveform within the frame, $\epsilon[n]$ should be identically zero.

The problem with this scheme is that, in general, the period of an acoustic source will not correspond to an integer number of samples. This problem has been encountered in many previous circumstances including the "long-term predictor" of traditional vocoders [3] and the delay lines at the heart of physical modeling music synthesis [4]. To get around this limitation, we employ a slightly more complicated filter to optimally remove the voice (figure 2, bottom),

$$
\epsilon[n]=x[n]-\sum_{i=-k}^{k} a_{i} * x[n-(\tau+i)] .
$$

or

$$
\mathbf{e}=\mathbf{x}-\mathbf{Z} \mathbf{a}
$$

where $\mathbf{e}_{i}=\epsilon[i], \mathbf{x}_{i}=x[i], \mathbf{Z}_{i, j}=x[i-(\tau+j)], \mathbf{a}_{j}=a[j]$; $i \epsilon[0, N-1]$ and $j \epsilon[-k, k]$. We used $k=3$ for a seven-coefficient filter as a more or less arbitrary compromise between computational complexity and flexibility of the cancelation filter.

The $a_{i}$ coefficients that optimally reduce the energy of $\epsilon[n]$ are found by the least squares solution,

$$
\hat{\mathbf{a}}=\left(\mathbf{Z}^{T} \mathbf{Z}\right)^{-1} \mathbf{Z}^{T} \mathbf{x}
$$

Having solved for these coefficients within each frame, we apply the filter to find the energy of the residual $\epsilon[n]$ within the frame,

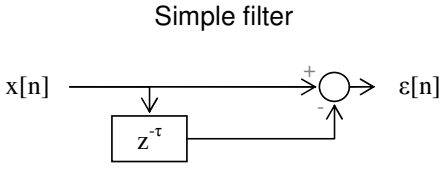

Sub-sample delay filter

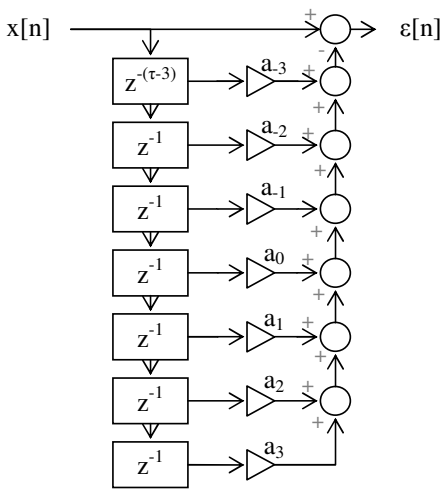

Figure 2: Optimal cancelation filters. Top: for signals with integer periodicities; bottom: a filter able to cancel non-integer periodicities.

then calculate the ratio of the residual energy to the energy of the original signal $x[n]$. In the case of a purely periodic signal whose period is a non-integer number of samples, we expect â to approximate an ideal fractional delay filter (sinc interpolator) which can exactly cancel the periodic signal, leading to a residual-to-original ratio close to zero. When the signal consists of many periodicities, only a small proportion of the energy will be canceled by eliminating just one dominant periodicity.

In frames consisting of "silence" (noise floor), however, a single spectral peak may account for a large proportion of the very small amount of energy. In this case, the optimal cancelation filter may also be able to remove a large proportion of the energy. To differentiate between silent frames and single voice frames, we added a value related to each frame's original energy as a second feature. To avoid any issues arising from global scaling of the original sound files, we normalized the entire waveform to make the 98th percentile of the short-time Fourier transform magnitude equal to 1 .

\subsection{Classifier}

We use the residual-to-original energy ratio and the normalized absolute energy as a two-dimensional feature and feed them to a simple Bayesian classifier to estimate the probability that each frame belongs to each of three classes - solo voice, multiple voices, and silence. We model the distribution of the features for each of these classes separately using a small amount of hand-labeled training data (see section 3). The normalized absolute energy is fit with a Gaussian in the $\log (\mathrm{dB})$ domain. The residual-to-original energy ratio, however, always lies between 0 and 1 , and is heavily skewed towards 0 in the solo class, and towards 1 in the multiple voice class. A Gaussian is thus a poor fit, and no simple transformation 
Raw classification

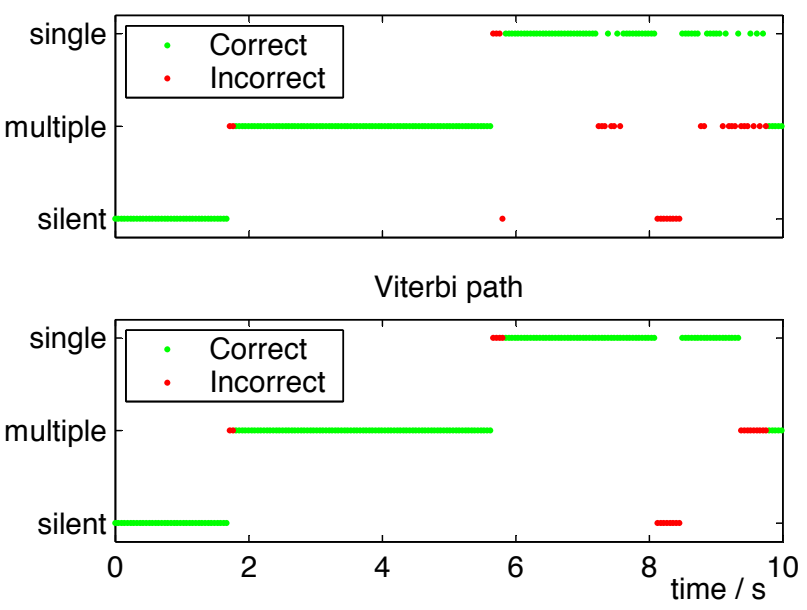

Figure 3: Example output from the cancelation-based classifier. Top pane shows raw frame-level results, bottom pane shows the result of HMM smoothing to remove rapid switching between states.

will make both these classes appear Gaussian. Instead, we model it with a Beta distribution for each category. The Beta distribution is defined over $[0,1]$ and has two parameters to fit both the mode and spread of the observed class-conditional values. We treat the two features as independent, so obtain the overall likelihood of a particular observation frame under each class as the product of the Gaussian and Beta fit to that class. Simple MAP classification then scales each likelihood by the prior of that class, and labels according to the largest resulting scaled posterior.

Independent classification of each time frame can result in rapid alternation between class labels, whereas real data changes state relatively infrequently. We build a simple three-state hidden Markov model (HMM) with transition probabilities set to match the empirical frame transition counts in the training data. We can then find the single most likely label sequence given this transition model and the class-dependent likelihoods with the Viterbi algorithm. (We used Kevin Murphy's Matlab implementation [5]. Figure 3 shows an example of label sequences before and after HMM smoothing, compared to the ground-truth labels.

To trade precision for recall, we can bias the model to generate more or fewer "solo" labels simply by scaling the solo model likelihood by a constant value. Smaller likelihoods for the "solo" class result in fewer, more confidently "solo" labels. In our application, assuming a very large underlying archive to search, we might be happy to accept a low recall (only a small portion of all possible solo regions are identified) in order to achieve a higher precision (nearly all of the identified regions are, in fact, solo regions).

\section{EXPERIMENTS}

\subsection{Data}

Our data set consisted of twenty 1 minutes samples that were handlabeled as silence, solo, or multiple voices. The samples were taken from a variety of professional folk and classical recordings. About $28 \%$ of the frames in the data set contained a solo voice.

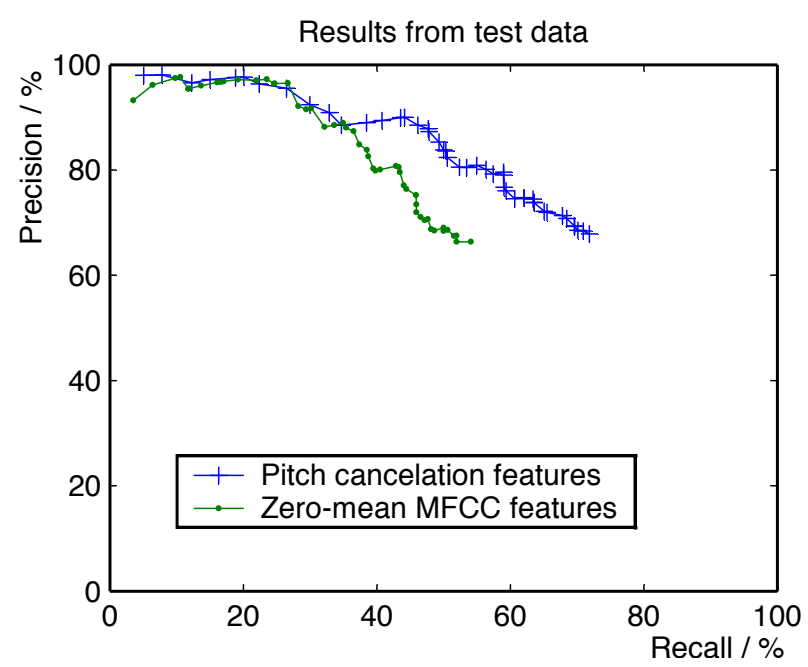

Figure 4: Solo voice detection precision/recall tradeoff for the two approaches.

The other $72 \%$ of data frames contained multiple voices or silence. Ten samples were used calculating the distribution and Viterbi path parameters. The remaining ten samples were used for testing.

\subsection{Baseline Classifier}

As mentioned in the introduction, we also implemented a 'generic' audio classifier based on the Mel-frequency cepstral coefficient feature vectors commonly used in speech recognition and that have also shown themselves very successful in many music classification tasks $[6,2]$. We used the first 13 cepstral coefficients and normalized their means to be zero within each track to eliminate any fixed filtering effects. The same Bayesian classifier structure was used, but in this case each of the three classes was fit by a fullcovariance multidimensional Gaussian. We used Netlab for this modeling [7].

\subsection{Results}

Figure 4 shows the results of the cancelation- and MFCC-based classifiers on all the test data combined. We obtained different precision/recall points by manipulating the single voice likelihood. Above about $90 \%$ precision, the MFCC and cancelation systems perform approximately equally. At lower precision levels, however, the cancelation algorithm has a much better recall. At $80 \%$ precision and below, the cancelation algorithm has at least $10 \%$ higher recall than the MFCC system.

The cancelation system also exhibits more consistent performance. When comparing the frame labeling accuracy on individual tracks in the test set, the standard deviation of the cancelation system performance was half that of the MFCC system. We suspect this is because the pitch cancelation algorithm has many fewer learned parameters (4 per class, compared to 104 per class for the MFCC) and thus is less susceptible to overfitting. 


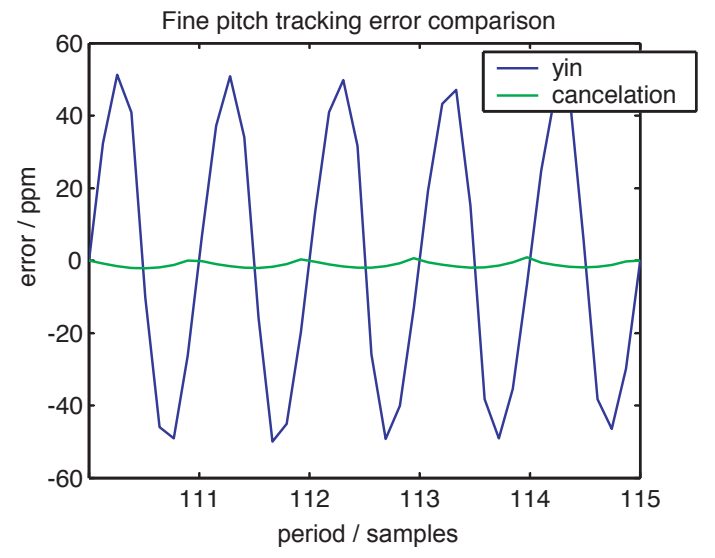

Figure 5: Comparison of fine pitch errors for synthetic signals showing the systematic bias resulting from YIN's quadratic interpolation.

\section{DISCUSSION AND CONCLUSIONS}

Although we resorted to the least-squares optimal FIR filter simply as a way to achieve precise fitting of non-integer-period signals, it is interesting to consider what we get as a result. The filter is optimized to minimize output energy, subject to the constraints that (a) the first value of the impulse response is 1 ; (b) the next $\tau-4$ are zero; and (c) the total length is $\tau+4$, where $\tau$ is our initial, coarse estimate of the dominant period. This filter is not constrained to include an exact unit-gain, linear-phase fractional delay, and in general energy will be minimized by a more complex response obeying these constraints. The seven free parameters of our system allow a certain error tolerance in the coarse period estimation as well as making it possible to match an ideal sync fractional delay more accurately, but they also permit a more complex range of solutions; solving for longer filter blocks would result in filters that deviate increasingly far from our intention of a simple comb canceling a single period.

If we can fit a pure delay to the delay path, that will give us a very accurate estimate of the dominant pitch in the signal i.e. a high-resolution pitch track. The delay can be simply estimated as a best slope fit to the unwrapped phase response of the Fourier transform of the optimal coefficients. In theory, this should give exact results when the signal is exactly periodic, rather than being biased to the integer sample delay periods of the baseline. As an example, figure 5 shows the error of pitch estimates obtained this way for synthetic, multiharmonic signals (of known period) compared to the state-of-the-art YIN algorithm [?] which uses a (heuristic) quadratic fit to estimate the peaks in an autocorrelationlike function between individual samples on the lag axis. Although the absolute errors are very small for both systems, we see that cancelation delivers significantly smaller errors in this case.

Another side effect is the residual signal, $\epsilon[n]$. For solo voices, this consists of the aperiodic component of the voice, which may be of interest e.g. for sinusoid+noise modeling, and/or may be useful for classifying the instrument. In ensemble recordings, the residual has effectively removed by comb filter the most energetic period close to the coarse estimate. This may go some way towards removing lead melodies e.g. to undo mixes. There may also be applications where the coarse period estimate comes from somewhere other than a first-stage autocorrelation. For instance, this kind of cancelation could form part of a score following system by locking in to a single periodicity in a mix whose approximate period and timing is known, but where refinements of both parameters is desired.

As discussed in the introduction, our goal was to find a way to accurately and automatically identify solo excerpts within a large music corpus, to collect training data for solo source models. We believe that the cancelation system is very suitable for this task, and our next step is to apply the system to a large music archive to see what we can find. The ability of the system to detect periodicity without a more detailed model of the particular voice to be found is both a strength and a weakness - it's useful to be able to detect solos for instruments not in the training set, but it means that the returns from the solo detection data mining will themselves need to be clustered and classified to build separate models for distinct instruments.

\section{REFERENCES}

[1] A. Klapuri, "Analysis of musical instrument sounds by source-filter-decay model," in Proc. IEEE ICASSP, Honolulu, HI, 2007, pp. I-53-56. [Online]. Available: http://www.cs. tut.fi/sgn/arg/klap/source.pdf

[2] A. Berenzweig and D. Ellis, "Locating singing voice segments within music signals," in Proc. IEEE Workshop on Apps. of Sig. Proc. to Audio and Acous., Mohonk, NY, October 2001, pp. 119-122. [Online]. Available: http: //www.ee.columbia.edu/ dpwe/pubs/waspaa01-singing.pdf

[3] B. Atal and M. Schroeder, "Predictive coding of speech signals and subjective error criteria," IEEE Transactions on Acoustics, Speech, and Signal Processing, vol. 27, no. 3, pp. 247-254, 1979.

[4] W. Putnam and J. Smith, "Design of fractional delay filters using convex optimization," Proceedings of the IEEE Workshop on Applications of Signal Processing to Audio and Acoustics, Oct. 1997.

[5] K. Murphy, "Hidden markov model (HMM) toolbox for matlab," Downloadable software, 2005. [Online]. Available: http://www.cs.ubc.ca/ murphyk/Software/HMM/hmm.html

[6] B. Logan, "Mel frequency cepstral coefficients for music modeling,", in Proc. International Symposium on Music Information Retrieval, Plymouth, 2000. [Online]. Available: http://ismir2000.ismir.net/papers/logan_paper.pdf

[7] I. Nabney, NETLAB: Algorithms for Pattern Recognition. London: Springer-Verlag London Ltd, 2004. [Online]. Available: http://www.ncrg.aston.ac.uk/netlab/ 